\title{
To pack or not to pack- A Randomized Clinical Trial comparing the Immediate Outcomes of Packed and Non Packed abscess wounds.
}

\author{
Bhuban Rijal, Prof. Vijay Kumar Sharma, Robin Bahadur Basnet, Gyan Pokhrel \\ National Academy of Medical Sciences, Kathmandu, Department of General Surgery.
}

Correspondence: Dr Bhuban Risal, National Academy of Medical Sciences, Kathmandu, Department of General Surgery

Email: bhubanrijal@yahoo.com

\begin{abstract}
Introduction: The objective of this study was to determine if there was any benefit from routine abscess packing after incision and drainage as compared to incision and drainage alone, as there seems to be emerging literature negating this practice.

Methods: A prospective randomized study incorporating abscess from different locations of the body, measuring less than $7 \mathrm{~cm}$, were taken. Incision and drainage was carried out under local or general anesthesia after routine preparation by the surgical resident under guidance of the surgical registrar. The subjects were then randomized into abscess cavity packing and non-packing groups with prescription for antibiotics, analgesics and narcotic analgesics. The subjects were followed up in surgical outpatient by the same resident, where the wound was examined, pain was noted for the duration using the VAS and number of narcotic analgesics taken were noted.

Results: Total of 92 patients was included divided equally in both groups. There was equality within the two groups regarding age, sex and location of abscess. The primary outcome, which was to find out the need for intervention in the first 48 hours after the procedure was compared and there was no significant difference in the interventions needed in the first 48 hours between the two groups ( $\mathrm{p}=$ 0.49 , difference in means $=.536,95 \% \mathrm{CI}=-2.14$ to 1.07 ). There was no difference in the overall pain perceived by the patients of the two groups, as the total patients who experienced pain were 77 , of which 41(53.2\%) were in the wound packed group and $36(46.8 \%)$ non-packed group, (difference in means $=0.783,95 \% \mathrm{CI}=0.40$ to $1.16, \mathrm{p}=0.75)$, but of the 19 patients who experienced severe pain, 15 were from the packed group and 4 were from the non-packed group $(p=0.02) .29$ patients from the packed group required narcotic analgesics whereas only 12 from the non-packed group required them $(\mathrm{p}=0.009,95 \% \mathrm{CI}=0.20$ to 0.58$)$.

Conclusion: Packing of the abscess cavity does not carry any added benefit as compared to Incision and Drainage alone and it causes more severe pain and discomfort to the patient with increased analgesic requirements.
\end{abstract}

Keywords: Abscess, Incision and Drainage, Packing and non-packing.

\section{Introduction}

Incision and Drainage of an abscess is the mainstay of treatment. The protocols of abscess drainage and post drainage treatment varies with different studies. These include differences in anesthetics used, irrigation of the cavity, packing of the cavity, culture of the abscess contains and antibiotic uses. ${ }^{1}$

Packing of the wound cavity has been a standard followed by physicians all around the world. Packing of the cavity is done primarily to prevent it from premature closure and 
to allow the collected material to come out. As per these recommendations, packing has been one of the main management steps in the procedure of incision and drainage of an abscess. However, more recent research has shown that these theories are not well supported by evidence. In one study, the healing time due to packing had been prolonged which led to more absence from working days than in non-packed wounds. ${ }^{2}$ This was further elaborated later, where there was evidence of improved healing and reduction of pain in patients who underwent perianal abscess drainage without packing as compared to packed wounds. ${ }^{3}$ Packing also has the disadvantage that it can lead to repeated visits to the hospital for packing change or removal due to soakage, increasing inconvenience caused to the patient. This can cause a lot of difficulties on the patient and cast further financial burden.

Although, there are some limited studies which state that packing of the abscess cavity can cause more discomfort, it is still being used by many physicians worldwide. This study compares the inconveniences caused by wound packing and compares the complications of the packed and non-packed wounds in the immediate post I\&D period.

\section{Methods}

A prospective randomized study of 102 adults who presented with an abscess in the surgical outpatient department of National Academy of Medical Sciences (NAMS), Bir Hospital, who underwent incision and drainage, were included in this study. An informed consent was provided to and obtained from each subject.

The surgery was conducted in Unit III General Surgery of NAMS, Bir Hospital from 2009-2011. All I\&D were performed by the Surgical Residents under the guidance of Surgical Registrar. All the participants were 18 years and older with a single abscess in the trunk, extremities and breast. Those who underwent I\&D were enrolled with a follow up after 48 hours and one week.

Exclusion Criteria were abscess larger than $7 \mathrm{~cm}$ in widest dimension, patients with comorbid conditions including Diabetes and HIV, immuno-suppressive states, steroid use, malignancy, undergoing CT and RT. Patients with perianal abscess, head neck and face abscess, chest wall abscess with extension into the thoracic cavity and abdominal abscess with intra-abdominal extension were excluded.

The study population was randomly divided into two groups. Group A were treated with I\&D and packing of the wound; while patients in group B were treated with I\&D alone. In both groups, local or general anesthesia was given according to the merit of the abscess. Adequate painting and draping was done and the abscess was incised and drained. The loculi were broken using a finger inserted to the cavity, the cavity wall were curetted and irrigated with normal saline. A gauge piece was packed and wound compressed if bleeding persisted. When adequate hemostasis was maintained, the patients were randomized according to odd and even numbers. In group A, a ribbon gauge was then soaked with povidone-iodine solution and packed in the abscess cavity. The wound was then closed with a gauge piece and tape. In group B, after securing complete hemostasis, the wound was covered with 2-3 pieces of gauze and tape without any pack left inside.

Both the groups received oral antibiotic, Cloxacillin $1 \mathrm{gm}$, Ibuprofen-Paracetamol tablets for pain relief with addition of weak opioid analgesic as required when needed. The patients were instructed not to change the dressing but to add an additional gauze if soakage was more than anticipated. All patients were asked to follow up on the surgical outpatient department on the third day. In the surgical OPD the dressing and pack were removed and the cavity inspected by the same resident who had initially carried out the procedure. Induration, erythema and fluctuation were noted. The patients' level of discomfort during the three days and the situation after the dressing were noted. The pain the patient had experienced during these three days were also noted using a visual analogue scale of 0 to $100 \mathrm{~mm}$. All the data were recorded in a preprinted score sheet and kept for analysis. The outcomes measured were the need for further intervention, which was described as need to extend the incision, probing of the cavity to break the loculi which had again formed, marked extension of erythema, induration and fluctuation needing hospitalization, additional antibiotic coverage. The other outcomes were the pain experienced, amount of pain killers used and the discomfort experienced by the patients.

\section{Results}

Eight patients were excluded from the study as they did not show up at follow up in 48 hours, 2 patients from the nonpacked group came to the emergency department the next day due to soakage whose dressings were changed and both were also excluded from the study.

Most of the patients were males and the mean age was 33 years. (Figure 1) Most of the abscesses were located in the gluteal region (26\%) followed by breast $(16 \%)$, thigh and forearm. (Table 1) 


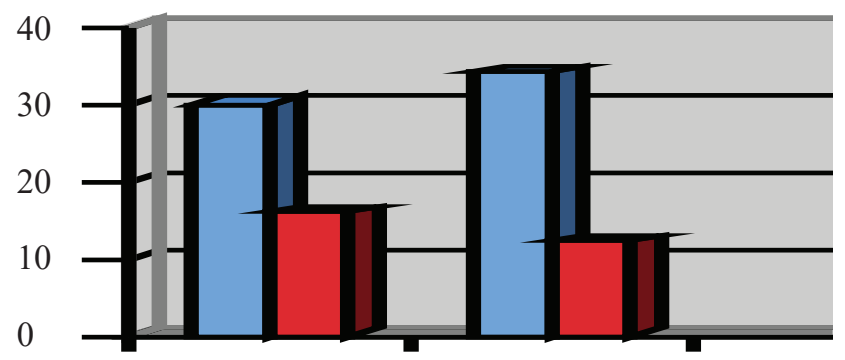

Packed Group

Table 1: Location of the abscess

$\begin{array}{ll}\text { Location } & \text { Number of Patients } \\ \text { Gluteal } & 24 \\ \text { Breast } & 15 \\ \text { Thigh } & 12 \\ \text { Forearm } & 11 \\ \text { Abdomen } & 8 \\ \text { Chest } & 7 \\ \text { Upper Arm } & 7 \\ \text { Back } & 3 \\ \text { Perineal } & 3 \\ \text { Others } & 2 \\ \text { Total } & 92\end{array}$

Table 2: Intervention needed in the both groups

\begin{tabular}{lll} 
Intervention & Packed & Non-packed \\
Extension of Incision & 4 & 3 \\
Irrigation of Cavity & 2 & 2 \\
Loculi Break up & 1 & 1 \\
Admission & 2 & 0 \\
Additional Antibiotics & 3 & 4 \\
\hline
\end{tabular}

Table 3: Pain assessment using VAS 1-100mm

$\begin{array}{lll}\text { Pain intensity } & \text { Packed } & \text { Non-packed } \\ \text { No pain (0-4mm) } & 5 & 10 \\ \text { Mild Pain (5-44mm) } & 7 & 22 \\ \text { Moderate Pain (45-74mm) } & 19 & 10 \\ \text { Severe Pain (75-100) } & 15 & 4\end{array}$

\section{Table 4: Use of pain Medications}

$\begin{array}{ccc}\text { Medications used in } 48 \text { hours } & \text { Packed } & \text { Non-packed } \\ \text { Weak Narcotic Analgesic } & 29 & 12\end{array}$

The primary outcome, which was to find out the need for intervention in the first 48 hours after the procedure was compared between the two groups of packed and nonpacked wounds. Total of 92 patients were followed up in the first 48 hours and of these 12 of the 46 patients from the packed group and 10 of the 46 patients from the non-packed group needed various interventions. (Table 2) There was no significant difference in the interventions needed in the first 48 hours between the two groups $(p=0.49$, difference in means $=.536,95 \% \mathrm{CI}=-2.14$ to 1.07$)$.

The pain reported by the patients that were recorded by the operating surgeon in the follow up was categorized as no pain VAS (0-4mm), mild pain (5-44mm), moderate pain (45$74 \mathrm{~mm})$ and severe pain $(75-100 \mathrm{~mm})$. While calculating the pain perceived by the packed and non-packed groups, we see that there is no difference in the overall pain perceived by the patients of the two groups, as the total patients who experienced pain were 77 , of which $41(53.2 \%)$ were in the wound packed group and $36(46.8 \%)$, (difference in means $=0.783,95 \% \mathrm{CI}=0.40$ to $1.16, \mathrm{p}=0.75)$. (Table 3 ) The intensity of pain in the two groups were different in three categories. 22 patients in the wound non-packed category developed mild pain as compared to 7 patients in the packed group, $(p=0.001)$. In the moderate pain group, 18 (29) patients in the packed group and 11(29) in the non-packed group experienced moderate pain $(\mathrm{p}=0.13)$. Of the 19 patients who experienced severe pain, 15 were from the packed group and 4 were from the non-packed group $(\mathrm{p}=0.02)$.

The requirement of narcotic analgesics were also analyzed in the two groups and 29(46) patients of the packed group and 12(46) patients of the non-packed group took additional narcotic analgesics in the first 48 hours ( $\mathrm{p}=0.009,95 \% \mathrm{CI}=0.20$ to 0.58 ). (Table 4 )

\section{Discussion}

Packing of the abscess cavity has always been included as a standard treatment protocol and has been followed by surgeons all around. Although, there is little evidence to back the purpose of abscess wound packing, which is mainly done so that the wound would not close prematurely and a dead space could be created which, would be pocket for pus and debris collection. ${ }^{4}$ The other benefit that has been proposed with wound packing is that with packing and removal, there was removal of the necrotic tissue from the cavity and walls.

This study has shown that even without packing the abscess cavity the need for secondary interventions and complications did not increase as compared to the packing group. The premature closure of the drainage incision and 
recollection in the cavity was not high, as it was presumed to be. In a similar study done by O'Malley and colleagues (2009), the incidence of secondary interventions were not different in both packed and non-packed abscess. ${ }^{5}$ Adequate primary drainage, wide incisions and dependent drainage techniques can all serve to give excellent results regardless of whether the wound is packed or not.

Pain after any incision and drainage procedure can cause inconvenience to the patients, which can limit mobilization of the affected part. This study not only tried to analyze the pain perceived by the patients but also tried to categorize the pain intensity. Although, the pain perceived in both groups were not different, the patients who had the I\&D wound packed, had a higher intensity of pain, as compared to the non-packed group. The non-packed group mainly had low intensity pain, which is obviously due to the procedure itself but higher intensity pain in the packed group was mostly due to the pressure effect of the packed material. Pain in any abscess is due to the inflammatory reaction and stretch of the surrounding tissue is one of the causes. While packing an abscess the stretch is not completely removed and the sensation still remains. This may be a cause for increased pain in our patients. There are some evidence of increased pain after packing of the abscess cavity but the intensity had not been observed individually in all these studies. ${ }^{6-7}$ The use of pain relievers after the procedure has been studied extensively. It is usually recommended that at least local anesthetic agents be used during the procedure and then use systemic analgesics after the procedure. ${ }^{8}$ In our practice, due to 48 hours of follow-up time, we prescribed narcotic analgesics to all patients and recommended them to use it as needed. The higher amount of narcotic analgesics used indicates that the patients who had their wounds packed experienced higher intensity of pain. As it was pointed out, that some pain relief can be achieved with the use of local anesthetic agents during the procedure, but there is no consensus in this issue. ${ }^{9}$

As we currently see no immediate decrease in the abscess formation and the treatment of this condition in the coming days, our strategy has to be one where there is minimal inconvenience to the patient and decrease the healing time. This can be obtained by not packing the wound routinely and using it only when very necessary.

\section{Conclusion}

Even though abscess packing is still widely used in practice, it has no added benefit as compared to abscesses' that are left unpacked. This study indicates the point that cavities that are not packed do not carry added risk for collection in the empty space, which may require secondary interventions. There is decrease in intensity of pain experienced by the patient, in the non-packed group and concomitantly, decrease in the use of analgesics prescribed. Non-packing of the abscess cavity may be recommended for abscess less than $7 \mathrm{~cm}$ in diameter but an institution wise protocol for Incision and Drainage has to be formulated.

\section{References}

1. May, L., Harter, K., Yadav, K., Strauss, R., Abualenain, J., Keim, A., \& Schmitz, G. (2012). Practice patterns and management strategies for purulent skin and soft-tissue infections in an urban academic ED. The American Journal of Emergency Medicine, 30(2), 302-310. doi:10.1016/j.ajem.2010.11.033

2. Stewart, M. P. M., Laing, M. R., \& Krukowski, Z. H. (1985). Treatment of acute abscesses by incision, curettage and primary suture without antibiotics: A controlled clinical trial. British Journal of Surgery, 72(1), 66-67. doi:10.1002/bjs.1800720125

3. Tonkin, D. M., Murphy, E., Brooke-Smith, M., Hollington, P., Rieger, N., Hockley, S., Wattchow, D. A. (2004). Perianal Abscess: A Pilot Study Comparing Packing With Nonpacking of the Abscess Cavity. Diseases of the Colon \& Rectum, 47(9), 15101514. doi:10.1007/s10350-004-0620-1

4. Butler, K.H. (2004). Incision and drainage. In: Roberts JR, Hedges JR, eds. Clinical Procedures in Emergency Medicine, 4th ed. pp726-7. Philadelphia, PA: Saunders

5. O’Malley, G. F., Dominici, P., Giraldo, P., Aguilera, E., Verma, M., Lares, C., Williams, E. (2009). Routine Packing of Simple Cutaneous Abscesses Is Painful and Probably Unnecessary. Academic Emergency Medicine, 16(5), 470-473. doi:10.1111/j.15532712.2009.00409.x

6. Kessler, D. O., Krantz, A., \& Mojica, M. (2012). Randomized Trial Comparing Wound Packing to No Wound Packing Following Incision and Drainage of Superficial Skin Abscesses in the Pediatric Emergency Department. Pediatric Emergency Care, 28(6), 514517. doi:10.1097/pec.0b013e3182587b20

7. Leinwand, M., Downing, M., Slater, D., Beck, M., Burton, K., \& Moyer, D. (2013). Incision and drainage of subcutaneous abscesses without the use of packing. Journal of Pediatric Surgery, 48(9), 19621965. doi:10.1016/j.jpedsurg.2013.01.027

8. Meislin H, Guisto J. (2010). Soft tissue infections, simple cutaneous abscesses. In: Marks J, Hockberger R, Walls R, eds. Rosen's Emergency Medicine:Concepts and Clinical Practice. 7th ed. pp1836-1847. Philadelphia, PA: Mosby Elsevier

9. Gillian, S. Goodwin, T. Singer, A. Kessler, Bhat, R. (2013). The Treatment of Cutaneous Abscesses Western J Emerg Med, 14(1). 2328 